\title{
PROKLA-Redaktion
}

\section{Zur Politischen Ökonomie des Wassers}

Den alten Gricchen galt Wasser neben Erde, Luft und Feuer als eines der »Vier Elcmente«, aus dcnen alles Seiende zusammengesetzt ist. Wasser ist die Grundlage allen Lebens. Wo Wasser zu finden war, ließen Mcnschen sich nieder, die Bändigung des Wassers ermöglichte die ersten Hochkulturen. Schon Herodot schrieb über den Glanz der Pharaonen, er sei »ein Geschenk des Nils « und Fernand Braudel arbeitete in seincr Kulturgeschichte der Mittelmeerregion die Bedeutung der hydrologischen und klimatischen Bedingungen für die soziale und ökonomische Entwicklung heraus. Wasser war auch ein entscheidendes Medium zur Erkundung der Welt und eine wunderbare Erleichterung des Transports. Zugleich ist Wasser in Gestalt von Flut- und Überschwemmungskatastrophen eine furchtbare Bedrohung. Aber nicht nur ein Zuviel auch ein Zuwenig an Wasser ist eine tödliche Gefahr: ausgedörrte Felder, vertrocknete Landstriche stellen das Überleben der Bevölkerung und ganzer Ökoysteme in Frage. Und erst recht entscheidet der Zustand des Wassers über Leben und Tod: $80 \%$ aller Krankheitsfälle (Cholera, Typhus, Diarrhö, Hepatitis) und ein Drittel aller Todesfälle in den Entwicklungsländern sind auf verseuchtes Wasser zurückzuführen.

Daß sich durch exzessive industrielle und private Vernutzung die Qualität des Wassers beständig verschlechtert, ist seit Jahren bekannt. Daß auch Flüsse versiegen und großflächige Seen (wie im Moment der größte Binnensee der Welt, der Aralsee) austrocknen können, dringt mitsamt den ökologisehen und sozioökonomischen Konsequenzen erst langsam ins Bewußtsein der Öffentlichkeit. Nicht nur Wasserverschmutzung, sondern auch Wasserknappheit wird in Zukunft selbst in bislang wasserreichen Regionen zu den alltäglichen Problemen gehören: Kalifornien mit seinen gigantischen Golfplätzen und Swimmingpools hat die Wasserknappheit ebenso kennengelernt wie das Rhein-Main-Gebiet. In Spanicn wurde bereits der »Wassernotstand « ausgerufen und zahlrcichc Staaten Afrikas erleben seit Jahren eine der cxtremsten Dürreperioden.

Die unterschiedliche Verfügung über Wasser spiegelt aber nicht einfach die geographische Ungleichverteilung der Wasservorräte wider, sie ist auch Ausdruck der ungleichen Verteilung politischer und ökonomischer Macht. Es macht also Sinn nach der politischen 
Ökonomie des Wassers zu fragen. Die zunehmende Wasserknappheit, die sich hierzulande bis jetzt vor allem in steigenden Wasserrechnungen bemerkbar machte, kann in anderen Regionen der Welt vielleicht schon in naher Zukunft kriegerische Auseinandersetzungen hervorbringen. Vor allem im israelischarabischen Konflikt ging es immer auch um die Verfügung über die begrenzten Wasserreserven der Region. Einen Überblick über Konfliktpotentiale, die zu einem »Krieg um Wasser führen können, gibt Ulrich Albrecht in seinem Beitrag.

Der Wasserkonflikt im Jordanbecken steht auch im Mittelpunkt des zweiten Artikels. Ines Dombrowski, Niels Gottschalk und Nadia Mazouz diskutieren, in welcher Weise eine die Kriterien des internationalen Rechts sowie inter- und intragenerationclle Beziehungen berücksichtigende »gerechte« Lösung dieses Konflikts überhaupt aussehen könnte.

Auswege aus der drohenden Wasserknappheit werden häufig nur unter technologischen Aspekten diskutiert. Es wird über die im ewigen Eis der Antarktis schlummernden Süsswasseressourcen ebenso nachgedacht, wie über den Ausbau der Meerwasseraufbereitung. Nicht berücksichtigt bleibt bei diesen technischen Utopien aber in der Regel das, was Hardin bereits vor Jahren als »tragedy of the commons « beschrieb: der individuell rationale (und das heißt unter kapitalistischen Bedingungen profitmaximierende) Umgang mit den »freien Gütern« birgt in sich die Tendenz zu ihrer Zerstörung. Für die Meere, eines der wichtigsten freien Güter, zeichnet Frank Biermann den Prozeß ihrer Aneignung durch den Menschen und damit auch ihrer Plünderung nach und beschreibt den mühsamen Weg einer politischen Regulierung des Umgangs mit dem Meer.

Der seit über einem Jahrzehnt vorherrschende neoliberale Diskurs hat allerdings auch zu entgegengesetzten Vorschlägen im Umgang mit den bisher freien Gütern geführt: statt politischer Regulierung wird Deregulierung und eine Ausweitung der Herrschaft des Marktes gefordert. In Großbritannien, wo in den 80er Jahren das neoliberale Credo besonders gläubige Anhänger fand, wurde eine Vielzahl traditionell »öffentlicher « Bereiche privatisiert - so auch die Wasserversorgungsbetricbe. Die keineswegs eindeutigen Folgen dieser Privatisierung werden von Simon Guy und Simon Marvin untersucht: während sich unter ökologischen Gcsichtspunkten Verbesserungen im Umgang mit dem Wasser feststellen ließen, führte dagegen die auf den Gewinn der Versorgungsunternehmen zielende Preisgestaltung bei einkommensschwachen Haushalten zu einem bislang unbekannten Phänomen: der »Wasserarmut«,

水水

Außerhalb des Schwerpunkts erscheint im vorliegenden Heft ein bereits im Editorial der Nr. 100 angekündigter Beitrag von Gilbert Ziebura. Er setzt die dort geführte Diskussion um eine »Ortsbestimmung« fort und diskutiert 
die Auswirkungen der Globalisierungstendenzen für eine links-alternative Politik. Damit wird eine schon in früheren Ausgaben der PROKLA (vgl. etwa die Beiträge in den Nummern 90, 92, 95 oder 97) geführte Debatte aufgenommen, die wir angesichts ihrer politischen Relevanz auch künftig weiterführen wollen.

Ebenfalls außerhalb des Heftschwerpunkts stehen die beiden letzten Bei- träge: Wulf Hopf diskutiert auf der Grundlage einer empirischen Studie die von rechtsradikalen Jugendlichen verwendeten Deutungsmuster gesellschaftlicher Wirklichkeit und Ingo Bode untersucht die vorweihnachtliche Streikbewegung in Frankreich, und die Reaktion der Linken vor dem Hintergrund einer Analyse unterschiedlicher Begründungen von Solidarität.

\section{PROKLA 103 (Juni 1996): Vom Gelde}

Geld regient die Welt, so heißt es jedentalls. Zentralbanken beispielsweise haben jede Menge Geld, da sie es selbst drucken können, und trotzdem scheint es im Zeitalter internationaler Geld- und Kapitalmảrkte nit ihrer Macht nicht weit her zu sein. In Sekunden werden auf diesen Märkten Milliarden, die niemand sehen oder anfassen kann, per Telefon und Computer rund um den Globus bewegt. Alles bloß eine virtuelle Realität oder die Macht, die die Welt im Innersten zusammenhalt? Die Okonomen wissen es nicht so genau: während der neoklassische Mainstream in Geld nur den Schleier iber den realwirtschaftlichen Verhältnissen sieht, erhebr der moderne Keynesianismus den Vermögensmarkt an die Spitze aller Märkte. Vielleicht kann die PROKL A erwas Licht in die Sache bringen.

\section{PROKLA 104 (September 1996): Universitäit}

Dab die Universitäten in der Krise sind, wird von Studenten, Protessoren und Politiken schon lange beklagt. Als Heilmittel werden Studiengebühren, Privatuniversitäten und kürzere Studienzeiten angeboten. DaR. Universitäten etwas mit Bildung zu tun haben könnten, gilt dagegen als hoftnungslos altmodisch. Aber was kann Universität heute tatsächlich noch bedeuten? Ist sie als Ausgangspunkt kritischen Denkens iberhaupt noch vorstellbar? Und wie sieht es mit der Universitätslandschaft anderer Lander aus? 